\title{
Automatic evaluation of human oocyte developmental potential from microscopy images
}

\author{
Denis Baručića ${ }^{\text {, Jan Kybic }}$, Olga Tepláb, Zinovij Topurkob ${ }^{\mathrm{b}}$, and Irena Kratochvílovác \\ ${ }^{a}$ Department of Cybernetics, Faculty of Electrical Engineering, Czech Technical University in \\ Prague, Czech Republic \\ ${ }^{\mathrm{b}}$ Department of Obstetrics and Gynecology, The First Faculty of Medicine and General \\ Teaching Hospital, Czech Republic \\ ${ }^{\mathrm{c}}$ Institute of Physics of the Czech Academy of Sciences, Czech Republic
}

\begin{abstract}
Infertility is becoming an issue for an increasing number of couples. The most common solution, in vitro fertilization, requires embryologists to carefully examine light microscopy images of human oocytes to determine their developmental potential. We propose an automatic system to improve the speed, repeatability, and accuracy of this process. We first localize individual oocytes and identify their principal components using CNN (U-Net) segmentation. Next, we calculate several descriptors based on geometry and texture. The final step is an SVM classifier. Both the segmentation and classification training is based on expert annotations. The presented approach leads to a classification accuracy of $70 \%$.
\end{abstract}

Keywords: human oocytes, fertilization, microscopy, classification, segmentation

\section{INTRODUCTION}

Infertility has been an issue for several years and is expected to grow further. Nowadays, the most common solution for an infertile couple is in vitro fertilization (IVF). One of the crucial steps is choosing the best oocytes to be fertilized, since, for practical, ethical, and legal reasons, it is not feasible to fertilize more than a few of them - and even fewer can be implanted.

The situation is easier when a patient's own oocytes are used and are, therefore, more readily available. In this case, the embryologists attempt to fertilize almost all collected oocytes that are not apparently damaged. The knowledge gained from these attempts can be used later to determine the a priori developmental potential of newly collected oocytes, reducing both the cost and the failure rate of the IVF. This is especially important when the oocytes come from a donor. In this case, each oocyte is very valuable, and it is important to determine its developmental potential reliably. Currently, this is performed by an expert who carefully examines the oocytes under a microscope. The selection process requires extensive experience, is time-consuming, and is done outside the optimal environment, so it is desirable to shorten it as much as possible.

In this work, we aim to replace this subjective process with an automatic evaluation of the developmental ability of oocytes from digitized light microscopy images to improve its speed, repeatability, and accuracy. We present a proof-of-concept solution on a small dataset, showing the viability of this approach.

\subsection{Previous work}

To the best of our knowledge, the task of fully automatic oocyte developmental potential assessment has not been addressed before. The closest work to ours is Manna et al., ${ }^{1}$ who feed LBP texture features to an ensemble of shallow neural networks and try to predict whether an oocyte or embryo (i.e., fertilized oocyte) will lead to birth. However, their approach requires manual segmentation, and obtaining the ground truth data takes much more time. Since multiple embryos are usually implanted, the outcome is only certain in the relatively few cases (namely $12^{1}$ ) when all or none of the implanted embryos lead to birth. Furthermore, many embryos are not implanted but frozen, and the pregnancy outcome might not be known for years or not at all. For this reason, here we have chosen a different classification target and decided to predict an embryo's ability to start the correct development, which can be determined relatively quickly and for all embryos. 

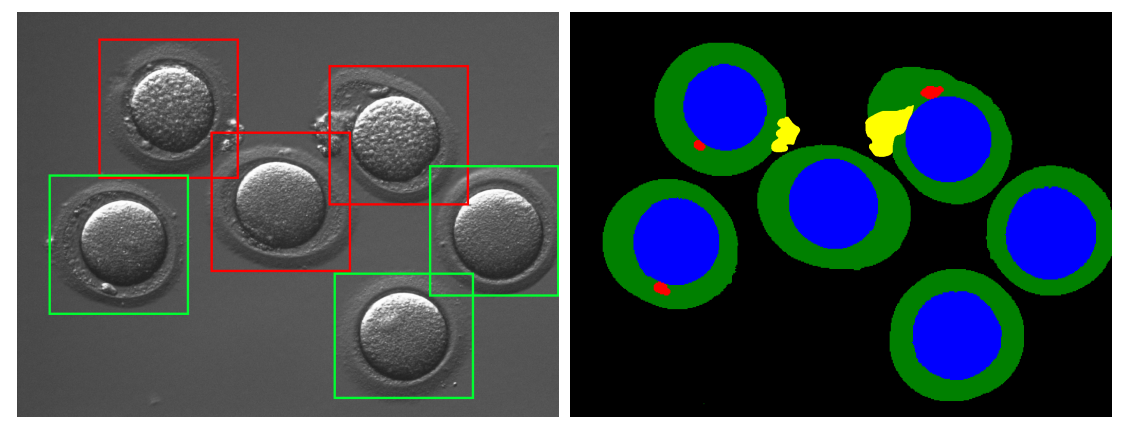

Figure 1. Example of an input image and the corresponding expert segmentation. The green and red frames denote viable and nonviable oocytes, respectively (left image). Classes background, cytoplasm, zona pellucida, polar body, and cumulus cells are denoted in black, blue, green, red, and yellow, respectively (right image).

Recently, deep learning has been used for oocyte segmentation. ${ }^{2,3}$ Other works analyzed microscopic images of embryos. ${ }^{4,5}$ An extensive database of 50000 embryo images allowed to train a model based on deep learning to classify the embryos into three quality classes. ${ }^{4}$ Raudonis et al. ${ }^{5}$ described a method for analyzing time-lapse sequences of embryo images.

Viswanath et al. ${ }^{6}$ classified swine cumulus oocyte complexes (i.e., oocytes with the cumulus cells, unlike in our data). The authors examined the number of cumulus cell layers and the homogeneity of the cytoplasm. A semi-automatic (snake) method was used for segmentation and random forests for the classification of oocytes. Pure texture analysis turned out to be useful for cytoplasm clustering ${ }^{7}$ or cytoplasm segmentation ${ }^{8}$.

\subsection{Proposed approach}

This paper describes a fully automatic approach to classify oocytes in light microscopy images (see Fig. 1) into two categories, viable and nonviable, where viable oocytes have a good potential of becoming well-developed embryos. We learn from subjective expert annotations of individual oocytes. Our approach consists of five consecutive stages - localization, extraction of individual oocytes, segmentation, feature extraction, and classification.

\section{DATA}

Our anonymized dataset consists of 34 grayscale images of groups of oocytes after cumulus cell denudation. Each image contains $1 \sim 7$ oocytes. The images of $1392 \times 1040$ pixels were acquired using Nikon Diaphot 300 inverted microscope, Eppendorf (Hamburg, Germany) micromanipulation system equipped with a thermoplate (Tokai Hit, Japan). The acquisition of the images was approved by the Ethics Committee of the General University Hospital, Prague, Czech Republic, on October 12, 2018, reference number 79/17.

The ground truth (GT) segmentations (see Fig. 1) were created using the GIMP graphical editor. We considered four classes: background, cytoplasm, zona pellucida, polar body, and cumulus cells. The individual oocytes were classified as viable or nonviable by the embryology expert (OT). Skipping incompletely visible oocytes yielded 50 viable and 53 nonviable oocytes. Furthermore, from observations of the development, we learned the true number of viable oocytes $y_{j}$ in each image $j$. This information is used to check the expert annotations in Sec. 4.5.

\section{METHOD}

Our method consists of five stages, explained in the following subsections. Fig. 2 gives a high-level overview.

\subsection{Oocyte localization}

We first perform a binary segmentation of cytoplasm versus the remaining classes because the cytoplasm is clearly distinguishable in the images. We use a U-Net ${ }^{9} \mathrm{CNN}$ with MobileNetV2 ${ }^{10}$ as the encoder, the standard mirroring decoder, and a pixel-wise softmax final layer. MobileNetV2 is a fast architecture with a relatively low number of parameters. The extra speed is desirable when the tool is deployed in a production environment. The 


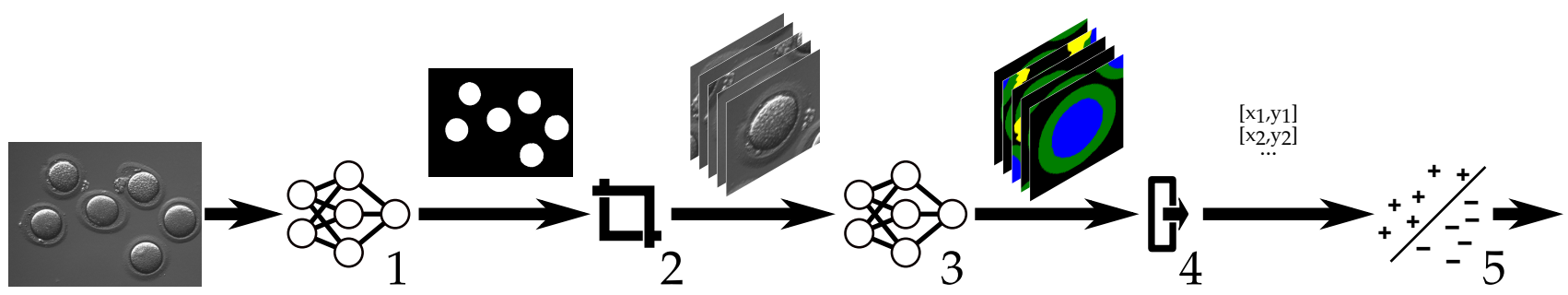

Figure 2. The classification pipeline. (1) Binary segmentation. (2) ROI extraction. (3) Five class segmentation. (4) Feature extraction. (5) Classification.

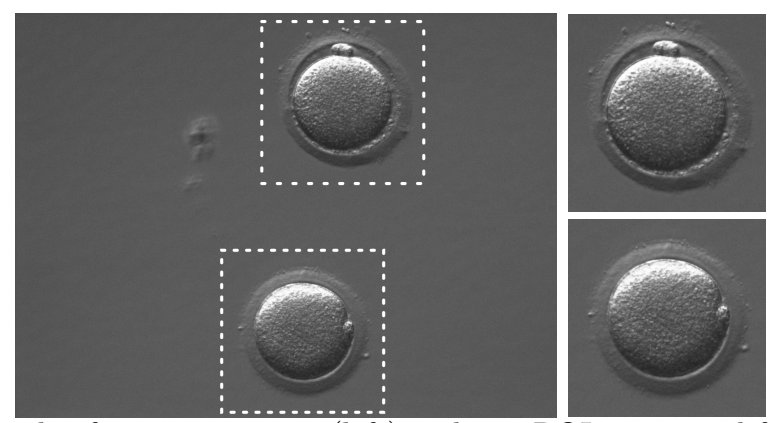

Figure 3. Example of an input image (left) and two ROIs extracted from the image.

network was trained for 500 epochs until convergence. We use the Dice loss function in combination with the ADAM optimizer (learning rate $10^{-4}$ ). To prevent overfitting, multiple data augmentation methods (shifting and rotation, contrast and brightness adjustments, and blurring) are applied to the training images during training.

Connected foreground components smaller than $10^{4}$ pixels are suppressed. The threshold was picked so that it does not rule out any viable oocytes, the area of which is always more than $4 \cdot 10^{4}$ pixels. Finally, regions of interest $(\mathrm{ROI})$ of size $416 \times 416$ are extracted from around the centers of gravity of the remaining foreground components (see Fig. 3).

\subsection{Oocyte segmentation}

Once the ROIs are extracted, they are segmented into the five classes described in Sec. 2 using another CNN. Since the polar body or cumulus cells classes are challenging to segment, we use a U-Net with the powerful ResNet50 architecture ${ }^{11}$ as the encoder, trained for 600 epochs, which was sufficient for convergence. The rest of the procedure is identical to Sec. 3.1. Example segmentations are shown in Fig. 4.

\subsection{Feature extraction}

Given the small number of available training images, we could not use deep learning for feature extraction. Instead, using the segmentation from the previous section (ROI) we compute for each oocyte the 24 features described below.

First, to handle the case where the ROI contains parts of several oocytes, we keep only the largest cytoplasm and zona pellucida components. We also suppress polar body components smaller than 500 pixels (for a bad oocyte, it is possible to have multiple polar body components, so we cannot just keep the largest).

Ellipses are fitted to the boundary of the cytoplasm class and to the outer boundary of the zona pellucida class by least squares fitting of the boundary pixels (see Fig. 5). We calculate the following features based on the cytoplasm ellipse:

- $\operatorname{mean}$ axis $\mu_{c}=\frac{a_{c}+b_{c}}{2}$,

- eccentricity $e_{c}=\sqrt{1-\frac{a_{c}^{2}}{b_{c}^{2}}}, a_{c} \geq b_{c}$,

- compactness $\gamma_{c}=\frac{a_{c} b_{c} \pi}{S_{c}}$, 

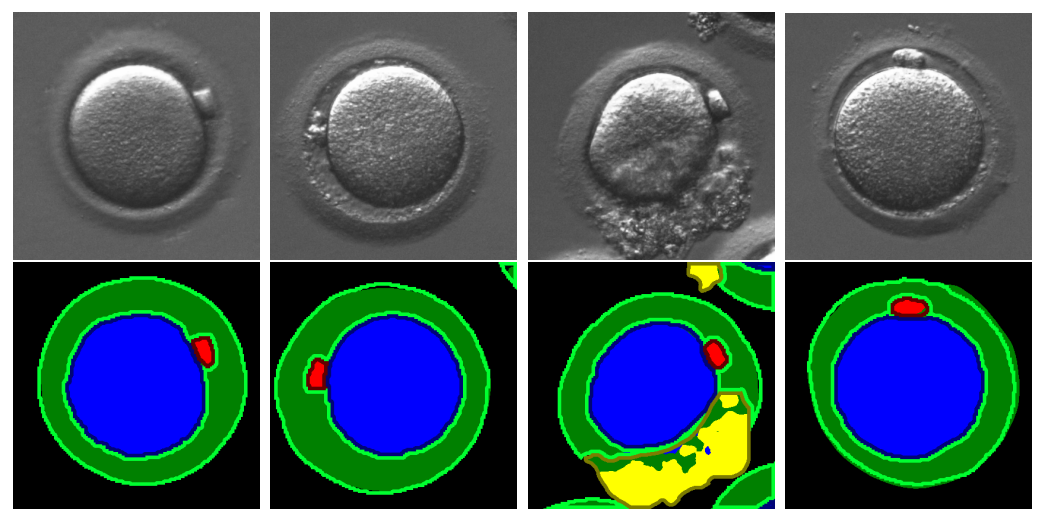

Figure 4. Four extracted ROIs in the top row and the corresponding predicted segmentations (black, green, blue, red and yellow denote background, zona pellucida, cytoplasm, polar body and cumules cells, respectively) and expert segmentations (outlined).
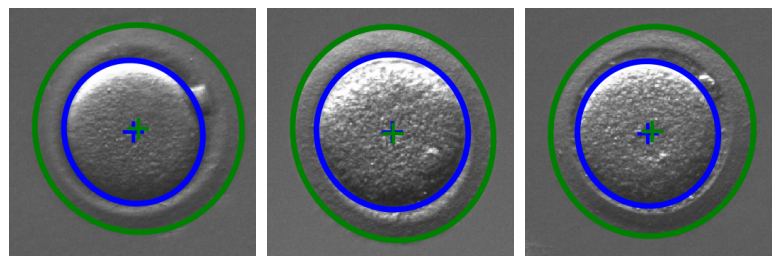

Figure 5. Fitted ellipses for cytoplasm (blue) and zona pellucida (green).

where $a_{c}, b_{c}$ are the estimated ellipse semi-axes, and $S_{c}$ is the area of the cytoplasm component. The features $\mu_{z}, e_{z}, \gamma_{z}$ are calculated similarly for the zona pellucida class. We also define the misalignment $m=\left\|\mathbf{c}_{c}-\mathbf{c}_{z}\right\|$, the Euclidean distance between the ellipse centers, and the ratio of the cytoplasm and zona pellucida areas, ${ }^{7}$ $r=\frac{S_{c}}{S_{z}}$.

Regarding polar bodies, two features are used: the number of connected components, $n_{p b}$, and the total area, $S_{p b}$. The presence of cumulus cells is not related to the oocyte quality but may influence the other features, hence we also calculate the total area of cumulus cells $S_{c c}$, which completes the 11 geometrical features.

The remaining 13 features describe the texture of the cytoplasm. ${ }^{1}$ The first 10 texture features are calculated efficiently from a three-level undecimated Haar wavelet transform ${ }^{12}$ and correspond to the energies in the low pass channel and 9 high frequency channels. The remaining three features are the mean, variance, and entropy of the pixel intensities in the cytoplasm.

\subsection{Oocyte classification}

For each oocyte, the extracted feature vector is normalized and fed into a binary classifier to produce a binary label, viable or nonviable. Several classifiers were tried with similar results. For the sake of space, only kernel SVM results are reported. The RBF SVM kernel with $\gamma=10^{-2}$ and the cost parameter $C=1.0$ were selected using stratified cross-validation and grid search on a training dataset of 83 randomly selected oocytes (ROIs). The remaining 20 ROIs were left for testing (Sec. 4.3). This yielded the mean validation accuracy $A_{\text {val }} \approx 74 \%$.

\section{RESULTS}

First, we evaluate the three stages of the pipeline separately. After that, we examine the significance of the designed features. Finally, we analyze the expert annotations.

\subsection{Oocyte localization evaluation}

Cross-validation was performed to evaluate the oocyte localization described in Sec. 3.1 using the 34 training images. For our data, the method worked perfectly. The number of detected oocytes was always equal to the ground truth and the localization error between the centers of gravity of the cytoplasm class, and the ground truth 


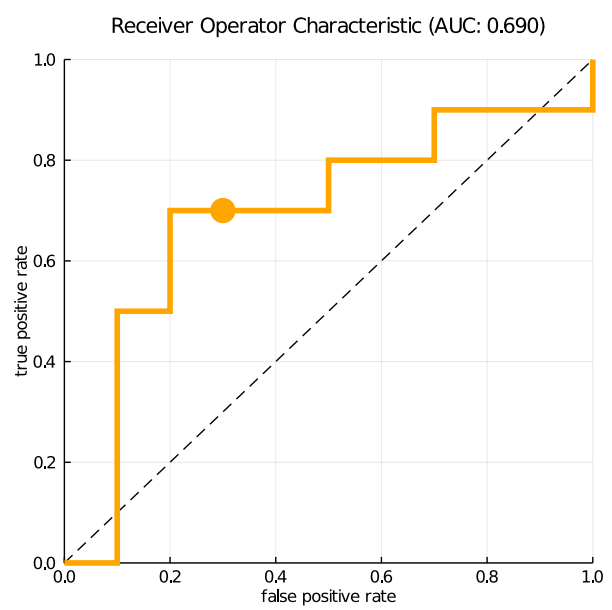

Figure 6. ROC curve obtained for the SVM classifier on the testing data. The dot represents our operating point.

cytoplasm segmentation was inferior to 10 pixels (corresponding to approximately $5 \%$ of the oocyte diameter) in $98 \%$ of cases.

\subsection{Oocyte segmentation evaluation}

The five-class oocyte segmentation (Sec. 3.2) was evaluated using a 10-fold cross-validation on the 103 ROIs, each approximately centered on one oocyte.

The Intersection over Union (IoU) metric computed over the folds for the cytoplasm, zona pellucida, polar body, and cumulus cells classes was $95.48 \%, 89.72 \%, 42.85 \%$, and $60.29 \%$, respectively. While cytoplasm and zona pellucida are segmented very well, the polar body class suffers from false detections and is more difficult to segment due to its small size. Although the cumulus cells segmentation performance is also far from perfect, it is mostly confused with background, which is not critical for our task (see Fig. 4).

\subsection{Oocyte classification evaluation}

The performance of the SVM from Sec. 3.4 to classify the oocytes as viable or not was evaluated using the 20 testing ROIs omitted during the training.

The classifier obtained the testing accuracy $A_{\text {test }}=70 \%$, sensitivity $S e_{\text {test }}=70 \%$, specificity $S p_{\text {test }}=70 \%$, precision $P_{\text {test }}=70 \%$, and the area under the ROC curve was $A U C_{\text {test }}=0.69$ (see Fig. 6).

\subsection{Feature significance}

To evaluate the features' significance, we ran a leave-one-out cross-validation on the whole dataset for different subsets of the features. Table 1 contains the results for four selected subsets. The most significant feature is the number of polar bodies $n_{p b}$. A viable oocyte contains a single polar body $\left(n_{p b}=1\right)$. However, in some images, the polar body may be hidden. Both the textural and geometrical features improve the classification accuracy, with a small additional improvement if using both.

Table 1. Accuracy achieved using four feature subsets. Each subset contains the number of polar bodies $n_{p b}$. The 13 texture features are denoted as "texture", and "geometry" denotes the remaining 10 features. The table shows the average accuracy computed using the leave-one-out procedure.

\begin{tabular}{lrr}
\hline features & number of features & accuracy \\
\hline$n_{p b}$ & 1 & 0.515 \\
$n_{p b}+$ texture & 14 & 0.738 \\
$n_{p b}+$ geometry & 11 & 0.767 \\
all & 24 & 0.777 \\
\hline
\end{tabular}




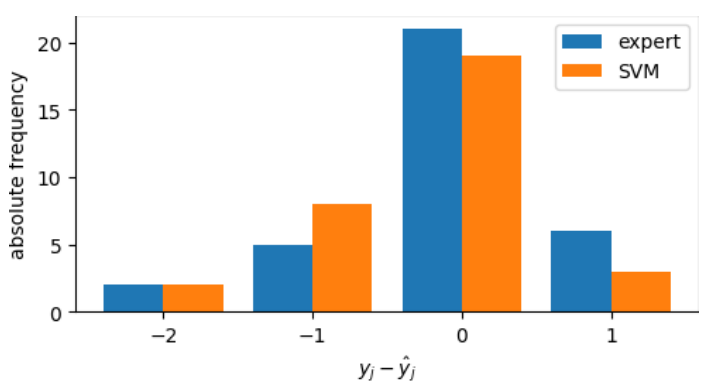

Figure 7. Histogram of annotation errors committed by a human expert and an SVM.

\subsection{Expert annotation evaluation}

The oocyte annotations created by the embryologist are used to train the classifier. Since these labels are subjective, we evaluate their expected accuracy using the reliably known number of viable oocytes $y_{j}$ as a reference.

Given image $j$, we denote $n_{j}$ the number of oocytes and $\hat{y}_{j}$ the number of oocytes labeled as viable by the expert. The mean absolute error of the expert MAE $=\frac{1}{N} \sum_{j=1}^{N}\left|y_{j}-\hat{y}_{j}\right|$, where $N$ is the number of images, was 0.44 with approximately 3 oocytes per image on the average. Fig. 7 displays a histogram of the differences in the number of viable oocytes per image for the annotations given by the expert and for those predicted by the leave-one-out cross-validation of our automatic method. The histogram reveals only a slight difference between the two annotations. The Kolmogorov-Smirnov statistic computed for the two histograms is $D=0.11$, which suggests no significant difference.

\section{DISCUSSION AND CONCLUSIONS}

In this paper, a proof-of-concept solution was proposed to automatically detect oocytes with good developmental potential and are therefore viable for fertilization. When interpreting the results, it is important to realize that the expert assessment of the oocyte quality from a single image is difficult and rather subjective. Our performance $(\mathrm{AUC}=0.69)$ is nevertheless comparable to the performance achieved by Manna et al. ${ }^{1}$ (AUC=0.68), who worked with the more reliable information of whether an oocyte leads to birth. The errors in the number of viable oocytes per image are also comparable to that of the expert. We expect our accuracy to improve further when more data is available. This should enable us to employ deep learning techniques instead of hand-crafted features, boosting the performance. We are also working on learning directly from the number of viable oocytes.

\section{ACKNOWLEDGMENTS}

The authors acknowledge the support of the OP VVV funded project "CZ.02.1.01/0.0/0.0/16_019/0000765 Research Center for Informatics" and the Grant Agency of the Czech Technical University in Prague, grant No. SGS20/170/OHK3/3T/13.

\section{REFERENCES}

[1] Manna, C. et al., "Artificial intelligence techniques for embryo and oocyte classification," Reproductive biomedicine online 26(1), 42-49 (2013).

[2] Firuzinia, S., Afzali, S. M., Ghasemian, F., and Mirroshandel, S. A., "A robust deep learning-based multiclass segmentation method for analyzing human metaphase II oocyte images," Computer Methods and Programs in Biomedicine 201, 105946 (2021).

[3] Targosz, A., Przystałka, P., Wiaderkiewicz, R., and Mrugacz, G., "Semantic segmentation of human oocyte images using deep neural networks," BioMedical Engineering OnLine 20(1), 1-26 (2021).

[4] Khosravi, P. et al., "Deep learning enables robust assessment and selection of human blastocysts after in vitro fertilization," NPJ Digital Medicine 2(1), 1-9 (2019). 
[5] Raudonis, V. et al., "Towards the automation of early-stage human embryo development detection," Biomedical Engineering OnLine 18(1), 1-20 (2019).

[6] Viswanath, P. S. et al., "Grading of mammalian cumulus oocyte complexes using machine learning for in vitro embryo culture," in [2016 IEEE-EMBS International Conference on Biomedical and Health Informatics (BHI)], 172-175, IEEE (2016).

[7] Basile, T. M. A. et al., "A texture-based image processing approach for the description of human oocyte cytoplasm," IEEE Transactions on Instrumentation and Measurement 59(10), 2591-2601 (2010).

[8] Caponetti, L. et al., "Cytoplasm image segmentation by spatial fuzzy clustering," in [International Workshop on Fuzzy Logic and Applications], 253-260, Springer (2011).

[9] Ronneberger, O. et al., "U-Net: Convolutional networks for biomedical image segmentation," in [International Conference on Medical Image Computing and Computer-Assisted Intervention], 234-241, Springer (2015).

[10] Sandler, M. et al., "MobileNetV2: Inverted residuals and linear bottlenecks," in [Proceedings of the IEEE Conference on Computer Vision and Pattern Recognition], 4510-4520 (2018).

[11] He, K. et al., "Deep residual learning for image recognition," in [Proceedings of the IEEE Conference on Computer Vision and Pattern Recognition], 770-778 (2016).

[12] Unser, M., "Texture classification and segmentation using wavelet frames," IEEE Transactions on Image Processing 4(11), 1549-1560 (1995). 